\title{
MARKETING ESTRATÉGICO ODONTOLÓGICO EN EL MARCO DEL COVID-19 EN AMBATO, ECUADOR
}

\author{
Andrés Sebastián Vasco Aguilera * \\ https://orcid.org/0000-0003-2567-9075 \\ Christian Andrés Barragan Ramírez** \\ https://orcid.org/0000-0001-8027-7883
}

\begin{abstract}
RECIBIDO: Julio 2021 / ACEPTADO: Septiembre 2021 / PUBLICADO: Enero 2022
\end{abstract}
Como citar: Vasco Aguilera, Andrés; Barragan Ramírez, Christian. (2022). Marketing estratégico odontológico en el marco del covid-19 en Ambato, Ecuador. Telos: revista de Estudios Interdisciplinarios en Ciencias Sociales, 24 (1), Venezuela. (Pp. 7-23).

DOI: www.doi.org/10.36390/telos 241.02

\section{RESUMEN}

En un contexto pandémico cargado de crisis socioeconómica, el campo de la odontología también ha tenido desafíos para prestar sus servicios, ya que atender a los pacientes implica altos riesgos de infección en los ambientes odontológicos; sin embargo, los compromisos con la sociedad les han conllevado a reconfigurar sus acciones, en concordancia con las necesidades del mercado. En ese marco, la presente investigación tuvo como objetivo determinar las estrategias de marketing implementadas por los centros especializados de odontología en el cantón de Ambato (Ecuador), para afrontar la crisis económica generada por el COVID-19. Para ello, se indagó sobre el marketing estratégico consultando las posturas de Lambín (2009), Kotler y Armstrong (2007) y Munuera y Rodríguez (2012), entre otros. Metodológicamente se acudió a un tipo de investigación aplicada-descriptiva, con un diseño de campo-transeccional. La población estuvo conformada por todos los centros especializados de odontología activos y con infraestructura física en el cantón de Ambato, siendo los agentes informantes los odontólogos que prestan sus servicios en los mencionados centros. La técnica para la recolección de datos fue la encuesta, mediante un cuestionario estructurado con escala likert, validado su contenido por cinco (5) expertos en mercadeo y se midió la confiabilidad mediante el coeficiente Alfa de Cronbach, el cual arrojó 0.99. Los resultados evidencian una tendencia positiva en la implementación de estrategias de marketing orientadas hacia la diversificación, el crecimiento y la competitividad. Se concluye que, los centros especializados de odontología desarrollan

\footnotetext{
* Ingeniero en petróleos. Estudiante de la Maestría en Administración de Empresas en la Pontifica Universidad Católica del Ecuador de Ambato. Ecuador. Correo Electrónico: asvasco@pucesa.edu.ec

** Ingeniero Comercial. Mención: Marketing. Magister en Administración de Empresas. Mención: Planeación. Doctorando en Ciencias Sociales. Mención Gerencia. Docente de la Pontificia Universidad Católica del Ecuador de Ambato. Ecuador. Correo Electrónico: cbarragan@pucesa.edu.ec
} 
estrategias de marketing para afrontar el entorno turbulento y poder desenvolverse en términos competitivos, acorde a las necesidades odontológicas de la sociedad local.

Palabras clave: Estrategias de marketing, diferenciación, crecimiento, competitividad, sector odontológico, COVID-19, Ecuador.

\title{
Strategic dental marketing within the framework of COVID-19 in Ambato, Ecuador
}

\begin{abstract}
In a pandemic context fraught with socioeconomic crisis, the field of dentistry has also had challenges in providing its services, since caring for patients implies high risks of infection in dental settings; However, commitments to society have led them to reconfigure their actions, in accordance with the needs of the market. In this framework, the present research aimed to determine the marketing strategies implemented by specialized dental centers in the canton of Ambato (Ecuador), to face the economic crisis generated by COVID-19. For this, strategic marketing was investigated by consulting the positions of Lambín (2009), Kotler and Armstrong (2007) and Munuera and Rodríguez (2012), among others. Methodologically, a type of applieddescriptive research was used, with a field-transectional design. The population was made up of all the specialized dental centers active and with physical infrastructure in the canton of Ambato, the informant agents being the dentists who provide their services in the aforementioned centers. The technique for data collection was the survey, using a structured questionnaire with a Likert scale, its content validated by five (5) marketing experts and reliability was measured using Cronbach's Alpha coefficient, which yielded 0.99. The results show a positive trend in the implementation of marketing strategies oriented towards diversification, growth and competitiveness. It is concluded that specialized dental centers develop marketing strategies to face the turbulent environment and to be able to function in competitive terms, according to the dental needs of the local society.
\end{abstract}

Keywords: Marketing strategies, differentiation, growth, competitiveness, dental sector, COVID19, Ecuador.

\section{Introducción.}

La creación de nuevas especialidades y subespecialidades en campo de la odontología, como: la endodoncia, ortodoncia, pediatría, rehabilitación oral, cirugía, entre otros, en el ámbito internacional, ha conllevado al desarrollo de nuevas tecnologías, técnicas y tratamientos en el campo de la estética y de la salud bucal. Para Dental Tribune Latin América (2017), su auge ha sido abrupto también en América Latina, tanto por odontólogos como por pacientes en México, Chile, Colombia y Brasil, dinamizando la economía, desde las grandes empresas fabricantes hasta vendedoras de equipamiento odontológico, convirtiéndolas en grandes compañías que cotizan en las diferentes bolsas de valores a nivel mundial.

Sin embargo, ese escenario no ha sido tan alentador en Ecuador, el cual se ha afianzado con el COVID-19, pues indican Moscoso-Moscoso et al. (2020, p. 808), que el mercado sectorial:

"se encuentra retraído y no ha podido tener el mismo desarrollo de ingresos y facturación que sus pares en otros países de la región. Esto se debe al pobre 
desempeño del sector, relacionado con la falta de información disponible para el consumidor ecuatoriano acerca de nuevos productos, soluciones y materiales, debido a que las casas comerciales no realizan campañas de marketing centradas en comunicar al consumidor los atributos de nuevos productos, y los beneficios que estos aportarían tanto para sus clínicas como para sus pacientes".

A pesar que "en Ecuador se presenta una alta prevalencia e incidencia de diferentes patologías bucales. Las enfermedades orales constituyen un problema de salud pública (...)" (Parise-Vasco et al., 2020, p. 327), y aunque ello se haya detectado en investigaciones científicas, señala la Superintendencia de Compañías, Valores y Seguros de Ecuador (2019), que en el año 2018, la facturación de las catorce empresas dentales más representativas del Ecuador alcanzó solamente $\$ 47$ millones de dólares y con la pandemia del COVID-19 en el país la situación no ha sido más alentadora.

Desde el año 2020, Ecuador ha estado inmerso en desequilibrios económicos, ello lo corrobora la Comisión Económica para América Latina y el Caribe (2020, p. 12) (CEPAL), ente que proyectó que "el crecimiento del producto interno bruto para Ecuador tienen estimaciones negativas (-6.5\%); y aunque se tiene previsto mejorías en el 2021, como señala Primicias (2021, p.1): "el Banco Mundial considera que el país experimentará una recuperación de 3,4\%", seguirán presentes efectos perjudiciales en todas las actividades económicas, especialmente en el sector comercio y servicios, los que sostienen principalmente la economía de ese país". Complementariamente, indica el Comité Empresarial Ecuatoriano (2020) que:

"La paralización del 70\% del sector productivo genera pérdidas diarias de USD 350 millones, la caída en las ventas diarias estaría en el orden los USD 150 millones, lo que denotaría una pérdida total de al menos USD 4.500 millones, en lo que dure la crisis, dejando sin oxígeno financiero a las empresas" (p. 1).

De esta forma, se puede afirmar que, la realidad ecuatoriana en el marco del COVID-19 se encuentra en un ambiente turbulento y de acuerdo a Useche et al. (2021, p. 4), está inserto en:

"una crisis sistémica-dinámica y con mucha incertidumbre, que arropa también el ámbito empresarial en Ecuador". Ello genera reducción de la demanda (nacional e internacional) y de sus ingresos, dificultades para acceder a insumos, reducción 0 falta de mano de obra, difícil acceso a financiamiento (...)".

Las dificultades personales, profesionales y empresariales, que está generando la pandemia COVID-19, han generado múltiples retos para las clínicas odontológicas y los profesionales que se desempeñan en ese campo. Entre esos retos se encuentra: Afianzar en la cultura organizacional la bioseguridad en las clínicas odontológicas, con la exigencia del uso de indumentaria de protección (guantes, mascarillas, bata, entre otros), tanto para los profesionales de la salud como para los pacientes, dado que el riesgo de infección en entornos dentales es alto.

Al respecto, Suárez Salgado et al. (2020, p. 6), explican que la "odontología, los pacientes y profesionales dentales, debido a la naturaleza de sus procedimientos, pueden estar expuestos a microorganismos patógenos, incluidos virus y bacterias que infectan la cavidad oral y el tracto respiratorio", ya que la atención al paciente es cara a cara, existe una elevada exposición a saliva, sangre u otros fluidos, ampliando la vulnerabilidad de los odontólogos y asistentes que prestan sus servicios a contagiarse. Sobre esto, Sigua-Rodríguez et al. (2020, p. 
1), afirma que "los estudios que hoy tenemos sobre la COVID-19 en la odontología son muy pocos (...), lo que dificulta la decisión sobre los enfoques y acciones que sean las más apropiadas para controlar su transmisión y limitar las posibles consecuencias".

Otro reto que se ha tenido que asumir en este campo de la salud, son las estrategias implementadas por el gobierno nacional ante Estado de Emergencia Sanitaria para minimizar los contagios masivos (Moreno, 2020), entre las estrategias implementadas se encuentran el aislamiento social, restricciones de horario de movilidad local, temporalidad de funcionamiento de determinados servicios, entre otros. Al respecto, El Universo (2021), señala que el Comité de Operaciones de Emergencia decidió que en el cantón de Ambato se estipulasen restricciones para la circulación vehicular en fechas y horarios establecidos, decidieron continuar con el semáforo epidemiológico, restricción de actividades económicas y prohibición de actividades físicas que generen aglomeraciones de personas.

Así que las organizaciones han tenido que modificar horarios, reconsiderar la cantidad de pacientes a atender diariamente, entre otros, lo cual ha implicado una fuerte contracción en la prestación de servicios y, por tanto, en los ingresos. Para el Arboleda, et al. (2020), ello generaría un conjunto de consecuencias desfavorables económicas y sociales, dado que al reducir la cantidad de ingresos, también se contrae la capacidad adquisitiva de las personas, pudiendo deprimir el aparato productivo y, con ello, disminuir la inversión, cierre de clínicas odontológicas, minimizar la cantidad de puestos de trabajos, hasta aumentara los niveles de desempleo; aspectos que se pueden mantener incluso después de la fase crítica sanitaria.

Esos retos y sus consecuencias exigen a las clínicas odontológicas en Ambato emplear sus fortalezas para afrontar un entorno pandémico, complejo e incierto, pero sobre todo cumplir con los compromisos administrativos y financieros los han motivado a actuar reactivamente e implementar estrategias de marketing para crecer, diferenciarse y ser competitivas; lo cual ha motivado a que en el objetivo de este artículo fue determinar las estrategias de marketing implementadas por los centros especializados de odontología en el cantón de Ambato (Ecuador), para afrontar la crisis económica generada por el COVID-19.

\section{Metodología}

Se implementó un tipo de investigación aplicada-descriptiva con un diseño de campotranseccional. La población estuvo conformada por 34 centros especializados de odontología, activos y con infraestructura física en el cantón de Ambato, donde laboran 131 profesionales en el área, registrados en el colegio de odontólogos de la provincia de Tungurahua. Por ser pocas organizaciones y tener acceso a las mismas se acudió a un censo poblacional. Para cuantificar la cantidad de agentes informantes (odontólogos) se empleó el muestreo aleatorio simple, arrojando 45 profesionales en el área mencionada.

La técnica para la recolección de datos fue la encuesta, mediante un cuestionario estructurado, conformado por 54 reactivos con escala likert, conformado con 5 alternativas de repuestas a: siempre (5); casi siempre (4); algunas veces (3); casi nunca (2) y nunca (1). El instrumento pasó por la etapa de fiabilidad, mediante la validado por contenido por cinco (5) expertos en mercadeo y la confiabilidad se midió con el coeficiente Alfa de Cronbach, arrojando 0.99 (confiable).

Para simplificar los resultados se creó una rúbrica que agrupa alternativas con valoración cuali-cuantitativa-positiva: 5,4 y 3 , respectivamente, mientras que se hace referencia a que no 
hay afinidad cuando las respuestas predominantes correspondan a las respuestas con ponderación cuali-cuantitativa negativa: 2 y 1, respectivamente, como se puede observar en el cuadro 1.

Cuadro 1

Rúbrica Cuali-cuantitativa

\begin{tabular}{|c|c|c|}
\hline $\begin{array}{l}\text { Valoración } \\
\text { Cuantitativa }\end{array}$ & Escala & $\begin{array}{l}\text { Valoración } \\
\text { Cualitativa }\end{array}$ \\
\hline 5 & Siempre & \multirow{3}{*}{ Positiva } \\
\hline 4 & Casi siempre & \\
\hline 3 & Algunas veces & \\
\hline 2 & Casi Nunca & \multirow[b]{2}{*}{ Negativa } \\
\hline 1 & Nunca & \\
\hline
\end{tabular}

Fuente: Elaboración propia.

Para la aplicación del instrumento se acudió a las bondades de las tecnologías de información y comunicación, usando la plataforma formularios de Google Forms, en los meses de marzo y mayo del año 2020, con la base de datos de correos electrónicos creada, gracias al apoyo de los centros especializados de odontología que facilitaron los correos electrónicos de los odontólogos, y se pudo enviar a esos profesionales el cuestionario de manera digital, lo cual facilitó el proceso de recopilación de los datos, así como la agrupación y tabulación de los datos cuantitativamente desde esa plataforma. Posteriormente, se identificaron las estrategias y acciones de marketing detectadas y se comparten las mismas de manera teórica y empírica, así como el análisis de los datos y su presentación en los siguientes apartados del documento.

\section{Estrategias de marketing}

Las estrategias empresariales han sido desarrollas ampliamente por expertos en el campo administrativo (Chandler, 1962; Ansoff, 1965; Miles y Snow, 1978; Mintzberg et al, 1998; Porter, 1980; Furrer et al., 2007; Whittington, 2003), pues científica, académica y empíricamente responden directamente a los intereses de las empresas, así como a los requerimientos del segmento de mercado donde se desenvuelven, para generar beneficios y crear valor económico que les permita superar a la competencia.

Desde este marco, en el campo de marketing también ha habido un desarrollo estratégico importante por investigadores, como: Porter (1985), Lambin et al. (2009), Munuera y Rodríguez (2012), Best (2007), Walker et al., (2005), Ferrel y Hartline (2012), Mullins et al. (2013) y Kotler y Armstrong (2007), entre otros, y todos coinciden en que son acciones que implementan las empresas para lograr un determinado objetivo de marketing, bien sea penetrar en un segmento de mercado, captar clientes, ampliar las cuotas de participación y según Muñiz (2012) permiten posicionar a las empresas en una situación altamente competitiva.

Es amplia la tipología de estrategias creadas en este campo; por lo que, se decidió de acuerdo a las características del entorno y del sector odontológico en un Ambato ante el COVID19 trabajar con las estrategias más amplias: Diversificación, crecimiento y competitividad. Entendiendo a las estrategias de diferenciación, de acuerdo a Munuera y Rodríguez (2012), como las acciones que se desarrollan para ampliar bien sea unidades de negocios, nuevas líneas productos, la entrada en nuevas actividades de mercado-producto, que pueden implicar 
cambios en su estructura administrativa, procesos organizacionales, entre otros. Para Best (2007), dependiendo de la sensibilidad del mercado, es preferible diferenciarse estableciendo precios bajos; sin embargo, considera que cuando el mercado así lo permita, es posible establecer diferencias marcadas que facilite a los clientes identificar las bondades del producto/servicio y en la marca.

En relación a las estrategias de crecimiento, Lambin et al. (2009), señalan que están direccionadas al incremento de la venta de los productos, a la participación del mercado, al beneficio y al tamaño de la organización; de igual manera, los clasifica en tres niveles diferentes: hacia dentro del mercado de referencia en el cual opera, hacia dentro de la cadena de abastecimiento y en las oportunidades por fuera del campo normal de actividades.

Al respecto, señalan Kotler y Armstrong (2007), que las empresas utilizan varias acciones para sostener un crecimiento rápido del mercado durante el mayor tiempo posible. Para Monferrer (2013), todas esas acciones buscan acelerar la participación en los mercados de manera ascendente, bien sea creciendo de manera intensiva o por integración, mediante la disminución de los precios del producto/servicio, en circunstancias específicas para atraer a más compradores; mejorando la calidad del producto/servicio; añadiendo nuevas características, técnicas y tecnología, así como acentuar la publicidad. En consonancia con los autores, Canals (2001), también entiende por crecimiento la expansión hacia nuevos mercados, tanto geográficos como de clientela. En términos generales desarrollan una ventaja estable y única, con respecto a la competencia en su entorno competitivo.

Por su parte, las estrategias competitivas se entienden como las acciones que pretenden crear valor y generar una posición defendible frente a la competencia (Porter, 1985), así como posicionar a una empresa para maximizar las capacidades que la distinguen de la competencia directa, tomando en consideración los esfuerzos de comercialización, producción, investigación y desarrollo, entre otros; y de acuerdo a Lambin (2009. p. 285), "crear cierta superioridad sobre sus competidores inmediatos".

Sobre las tipologías desarrolladas, Miles y Snow (1978), Porter (1980), Miller (1987) y Kottler y Singh (1981), entre otros, coinciden en que estas estrategias tienen como objetivo central desarrollar una ventaja competitiva de las empresas, bien sea mediante acciones prospectoras, defensivas, analizadoras, reactivas, de líder, de especialista, y defensivas ante el conjunto de fuerzas que existen en el mercado, para mantener una posición distintiva, ventajosa y beneficiosa en el segmento de mercado donde se desenvuelven.

La diversidad de la literatura sobre las estrategias de marketing y sus tipologías ofrecen un abanico de posibilidades a las clínicas odontológicas de ser empleadas, de acuerdo a las características del sector odontológico en Ambato, pues en términos generales todas las empresas tienen similares características, aunque se distinguen en sus capacidades, tecnologías y actualizaciones. Ello permite determinar las estrategias de marketing predominantes y comunes para responder a las necesidades de la sociedad cantonal ante el COVID-19 y mantener su carácter competitivo, de crecimiento, diversificación y superación en el mercado local.

\section{Estrategias de Marketing Odontológicas en Ambato}

Los centros especializados de odontología en el Cantón de Ambato han venido implementando un conjunto de estrategias de marketing, para intentar paliar la crisis económicas 
en la nación y la región, las cuales han estado centradas en la diferenciación, crecimiento y competitividad de las mencionadas organizaciones, que en conjunto "tienen como principal interés asignar y coordinar efectivamente los recursos y actividades para alcanzar las metas de la compañía, dentro de un mercado específico" (Walker et al., 2007, p.42). Las acciones encontradas sobre ese trío de estrategias se presentan a continuación:

\section{a) Estrategias de diferenciación odontológicas en Ambato.}

Al recopilar los datos y tabular los mismos, se encontró que los centros especializados de odontología implementan estrategias de diferenciación, para procurar bajar los costos. En tal sentido, el $86,66 \%$ de los encuestados manifestó que vigilan los costos de los servicios y productos que ofrecen, lo cual permite que el $76,66 \%$ considere que sus precios sean más bajos en comparación a la competencia. Esa tendencia positiva evidencia que se ha prestado atención a controlar los costos para poder ofrecer precios, acorde a la capacidad adquisitiva de la población. Para Molina (2003), con el control de costos se pueden "(...) resolver múltiples problemas de gestión que existen en todo tipo de organización, puesto que están orientadas específicamente a optimizar el uso de los recursos de la empresa para alcanzar un objetivo: la competitividad".

Otra acción de diferenciación se ha orientado a crear y mantener cualidades distintivas que les permita marcar diferencias con la competencia; expresando el $96,66 \%$ que sus servicios tienen características que a los pacientes les es fácil identificar y diferenciar de la competencia, haciendo particular énfasis en que la calidad $(100 \%)$ del servicio sea superior a la prestada por otros odontólogos y centros especializados de odontología. Al respecto, señala Best (2009, p.215) que:

"En aquellos casos en los que la diferenciación sea posible y valorada por los clientes, la empresa dispone de otras alternativas. En aquellos casos en los que una empresa disponga de diferencias competitivas en el producto, en el servicio, en la imagen de marca, que sean significativas y posteriormente valoradas por su público objetivo, la empresa dispondrá del potencial de crear una posición de producto más atractiva que la competencia. Con independencia de la estrategia de diferenciación de producto decidida, el objetivo es proporcionar al cliente un valor superior al que ofrece la competencia".

Con estas acciones se "intenta garantizar el encaje estratégico donde se puede hacer un buen uso de su conocimiento sobre productos y sus destrezas de marketing que emplea de manera eficaz" (Medina et al., 2012, p. 58). Los hallazgos compartidos permiten afirmar que las acciones de diferenciación empleadas se han enfocado en distinguirse de otros profesionales odontólogos, destacando la imagen de un servicio único, sin pretender buscar o explotar nichos de mercado y tampoco intentar ampliar sus operaciones hacia otros negocios conexos a la actividad de salud. Sobre esta estrategia, $66,66 \%$ respondió de manera negativa, indicando que no tienen interés en realizar acciones de integración vertical, pues su atención no está centrada en controlar sus fuentes de suministros y tampoco en mejorar su rentabilidad interviniendo en diferentes actividades de importancia estratégica para las organizaciones en estudio.

\section{b) Estrategias de Crecimiento odontológicas en Ambato.}


A pesar de las dificultades socioeconómicas que experimenta la nación, los centros especializados de odontología han dirigido sus recursos y esfuerzos a intentar crecer localmente; en tal sentido, han empleado de manera predominante estrategias de crecimiento intensivo, mediante un conjunto de acciones orientadas al desarrollo de nuevas técnicas odontológicas $(96,66 \%)$, introducir nuevas generaciones de insumos odontológicos (95,66\%), disponibilidad de profesionales especializados con experiencia y dominio de competencias/habilidades en diversas ramas de la odontología, e invierten en nuevas tecnologías (100\%), y para invertir $86,66 \%$ manifestó afirmativamente que han empleado acuerdos de cooperación, así como alianzas estratégicas, facilitando la búsqueda de recursos para acceder a tecnología de punta a menor costo, que de acuerdo a Ariño (2007, p. 44), "permite acceder a recursos de los que la empresa carece y que, además, le resultaría muy costoso obtener de otra manera, sean estos recursos similares o complementarios a los que ya posee" y adicionalmente, "(...) crea un valor que no podrán desarrollar si actuaran de forma independiente" (Hitt et al., 2008, p.271).

Estas acciones estimulan a la actualización de conocimientos, acorde al desarrollo tecnológico, pues saben que el manejo de tecnología actualizada es un aspecto diferenciador de la competencia y eslabón de crecimiento sectorial, en concordancia con los avances científicos/técnicos y nuevas tendencias en ese campo de la salud en el ámbito internacional.

Con estas acciones de crecimiento, señalan Munuera y Rodríguez (2012), que se generan una serie de ventajas para la empresa, ya que los clientes se sienten atraídos por la imagen de una empresa que se encuentra a la vanguardia, ofreciendo tecnología vigente y habilidades de los profesionales actualizados; asimismo, los empleados y directivos se sienten orgullosos de trabajar en una empresa dinámica, dispuesta a invertir en pro de la salud de la población, lo cual se refleja en la calidad del servicio y en la imagen corporativa.

Otro tipo de acciones de crecimiento encontrada se enfoca en la penetración de mercado, mediante el incremento de la cuota de mercado. Al respecto, el 100\% de los encuestados respondió afirmativamente que: con frecuencia los centros de especialización de odontología publican nuevas promociones para estimular la frecuencia de la demanda de los servicios e informar sobre las bondades de las organizaciones de salud en estudio. Así mismo, la totalidad de los encuestados (100\%) manifestó que se esfuerzan en atraer al mercado local, ofreciendo precios accesibles ( $82 \%$ ) en concordancia con las necesidades de la población y atentos a que sean equiparables 0 inferiores a la competencia.

Con esta estrategia se busca captar más clientes/pacientes y ampliar la cobertura del mercado de las organizaciones en el sector de salud donde se desenvuelven, pues de acuerdo a Arellano (2010, p. 338), "cuanto mayor sea la penetración de mercado de la empresa, mayor será la generación de liquidez del producto/servicio".

Las acciones comunicacionales han tenido rol protagónico en tiempos de pandemia, para estimular la demanda de los servicios se han enfocado mantener una intensa publicidad digital, a fin de posicionar los servicios en el mercado local (73,33\%), es decir, en el cantón de Ambato, sin tener intensiones de ampliación geográfica, bien sea como sucursal o extensión a otros cantones o provincias del país, así lo expresó el $70 \%$ de los encuestados. Los medios de comunicación más utilizados para ofrecer los servicios de odontología se avocaron a las redes sociales $(100 \%)$, informando la variedad tanto de servicios como de productos disponibles, persuadiendo al público meta sobre las ofertas y promociones, así como la temporalidad de las 
mismas y recordando a los pacientes las ventajas y beneficios que implica atender su salud bucal en esos centros especializados.

Al respecto, coinciden Aguilera-Castro et al. (2017), y Real et al. (2018), que en tiempos de pandemia el uso de la tecnología puede significar un aumento en la modernización y agilización de la relación con los clientes; destacando la alta demanda que han tenido las aplicaciones tecnológicas, pues según Demuner Flores (2021), con ellas se siguen ampliando las posibilidades de comunicación y relaciones directas con clientes y proveedores. Con una mirada estratégica desde el marketing, coinciden Villagómez y Acosta (2020), en que los medios de comunicación digitales son la vía para aplicar las estrategias de marketing, ya que facilitan la interacción con los clientes actuales y potenciales, así como coadyuva difundir de manera masiva sus propiedades, variedad de bienes y servicios.

Definitivamente que la comunicación en el marketing ha tomado desde el año 2020 gran ímpetu; para Tarazona (2020), y Hill (2020), con la pandemia se ha dado un giro trascendental ante las necesidades de una población, obligada al confinamiento temporal, conllevando también al cierre de diversas actividades, y para evitar el colapso, se emplearon rápidamente nuevas formas de relaciones, que los autores catalogan como un tsunami de innovación comunicacional, también complejo y con grandes desafíos a afrontar.

A manera de síntesis, se ha elaborado el grafico 1 para agrupar todas las acciones desarrolladas que corresponde a la estrategia de crecimiento intensivo de los mencionados centros de salud, en el cual se detecta el desarrollo y actualización de las competencias, inversiones en nuevas tecnologías, y actualización de técnicas odontológicas, entre otros.

\section{Gráfico 1}

Acciones de crecimiento Intensivo

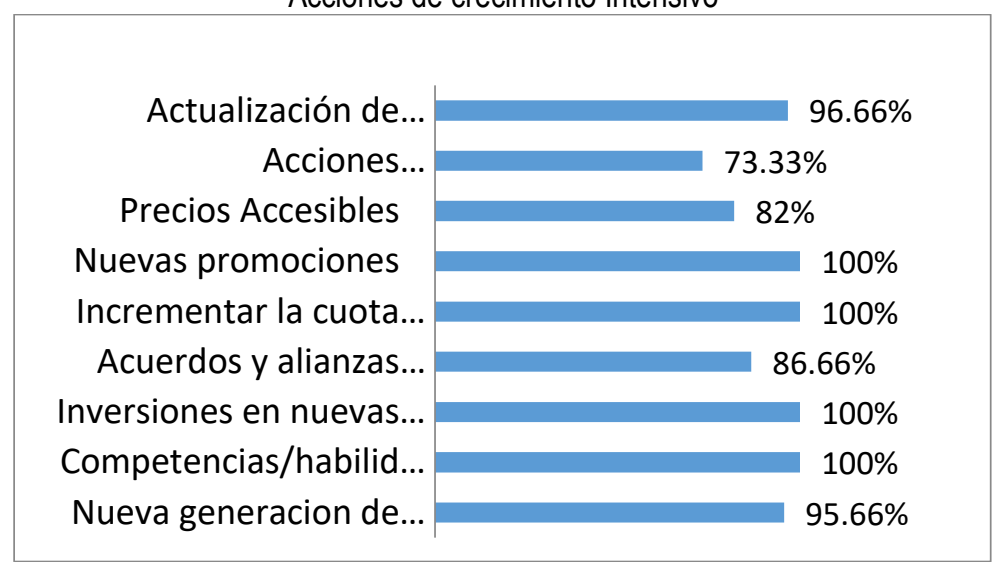

Fuente: Elaboración propia.

Es común que haya tantas acciones desplegadas en relación a las estrategias de crecimiento, ya que este tipo de estrategias en términos generales procura impulsar en el mercado las relaciones de los centros especializados de odontología con otras entidades y 
pacientes con necesidades de salud odontológica en el mercado, en pro de la rentabilidad y posicionarse en la mente de los usuarios.

Así como el mercado no volverá a ser el mismo, tampoco serán igual las estrategias de crecimiento tradicionales que venían empleando estas organizaciones antes de la pandemia y en la misma. Las estrategias de crecimiento aplicadas evidencian que quedarse estáticas no ha sido una opción porque los contextos demandan adaptación oportuna, y crecer exige cambiar hasta el enfoque de marketing acorde a las nuevas pautas del mercado.

\section{c) Estrategias de competitividad odontológicas en Ambato}

Para Navarro (2021, p. 11), "la historia ha demostrado que las crisis también generan oportunidades y, por tanto, la COVID-19 podría ser un golpe de realidad para reflexionar y repensar diferentes cuestiones", entre ellas, la competitividad del siglo XXI de estas organizaciones de salud, pues sus ansias de progresar, consolidarse en el mercado y obtener resultados económicos superiores a las competencia persisten, ubicándolas en una posición de mercado sostenida o ampliada en el segmento donde se desenvuelve.

Desde la persistencia competitiva, estas organizaciones emplearon estrategias de competitividad, a través de acciones prospectoras, las cuales son "apropiadas en ambientes inestables y rápidamente cambiantes ocasionados por las nuevas tecnologías y los cambios en las necesidades de los clientes, o ambos" (Walker et al., 2007, p. 232). Sobre ellas, los encuestados expresaron que estas organizaciones impulsan con frecuencia nuevas oportunidades de innovación (100\%) y nuevos modelos de negocios (100\%) porque saben que es un trabajo recurrente fortalecer su posición en el mercado, desde la innovación y nuevos modelos de negocios. Estos resultados se encuentran en concordancia con Cordero et al. (2003), demostrando al mercado que se poseen ventajas sobre la competencia, así como las capacidades para extender su participación en el mercado local de manera beneficiosa económicamente, que permita su crecimiento en un entorno cambiante.

De igual forma, la totalidad de los encuestados (100\%) hacia una tendencia positiva, estuvo de acuerdo en que estas organizaciones aplican acciones analizadoras, defensivas y reactivas. Las acciones analizadoras, empleadas se basan en la creación de nuevos servicios para evitar ser superados por sus competidores (otros centros odontológicos), los cuales tienen como criterios la calidad, bajos costos y precios inferiores a la competencia. Para Walker et al. (2007, p. 234), esta estrategia es:

"(...) un híbrido. Por una parte, están ocupados en defender -mediante bajos costos 0 diferenciación de calidad o servicio- una posición de participación fuerte en uno o más mercados de producto establecido. Al mismo tiempo, desarrollan nuevos productos para evitar que los competidores le salten por encima con productos tecnológicamente más avanzados, o que se quede atrás en segmentos de aplicación recién desarrollados dentro del mercado".

Mientras que, las acciones defensivas aplicadas se han orientado en mantener los costos por debajo de la competencia (100\%), para ofrecer un mejor precio de mercado, con ello se evidencia la posición de Miles et al. (2003), que con este tipo de acciones no se busca entrar en 
nuevos mercados, sino obtener incrementos económicos en sus operaciones y mantener estabilidad en los mismos.

Con respecto a las acciones reactivas, la totalidad de los encuestados respondieron que las organizaciones tienen rápidas respuestas ante los cambios de la economía nacional, las necesidades y demandas del mercado local. Para Munuera y Rodríguez (2012), eso es propio y característico de una estrategia reactiva no responder tardíamente a los cambios que están ocurriendo, es decir, tomar decisiones basadas en las presiones del entorno. Esas acciones reactivas se corresponden con las acciones de liderazgo, las cuales también estuvieron presentes entre las respuestas de los encuestados. Todos los agentes informantes (100\%) manifestaron que los centros de odontología se han esforzado en desarrollar acciones para captar nuevos usuarios, es decir, atraer a los consumidores que no lo conocen o que se resisten disfrutar del servicio/producto.

Estos centros también han introducido nuevos servicios, para fortalecer la posición competitiva y diferenciarse de la competencia. Este comportamiento se corresponde con la posición de búsqueda de liderazgo de Kotler y Amstrong (2007, p.703), quienes indican que para ser líder se requiere de diferentes acciones para "(...) aumentar la demanda total, proteger su participación presente en el mercado y tratar de expandir aún más su parte en el mercado, incluso cuando el tamaño del mercado sea constante".

Al igual que las acciones prospectoras y de liderazgo, las retadoras también se encontraron en el desenvolvimiento del marketing odontológico. En tal sentido, las organizaciones en estudio emplean recursos y actividades procurando superar las fortalezas de la competencia local (98\%); así mismo, los encuestados manifestaron que los centros de especialización de odontología han estado atentos a la conducta de marketing de la competencia (90\%) y han ampliado la visión de sus servicios, atendiendo simultáneamente a pequeños grupos no atendidos en el mercado y a pacientes tradicionales $(75 \%)$ individuales y corporativos.

Si bien es cierto que todas las respuestas de las estrategias competitivas tienen tendencias positivas, se está en concordancia con Rubio y Baz (2015), en que la competitividad de cada organización depende de la flexibilidad de sus estructuras internas, es decir, de su resiliencia organizacional, capacidad de aumentar las ventas en mayor proporción que sus competidores y generar valor al servicio demandado, que en este caso obedece a la odontología, destacando la ventaja competitiva en el sector, mediante las acciones antes explicadas y que se resumen en el grafico 2.

Gráfico 2

Acciones Competitivas 


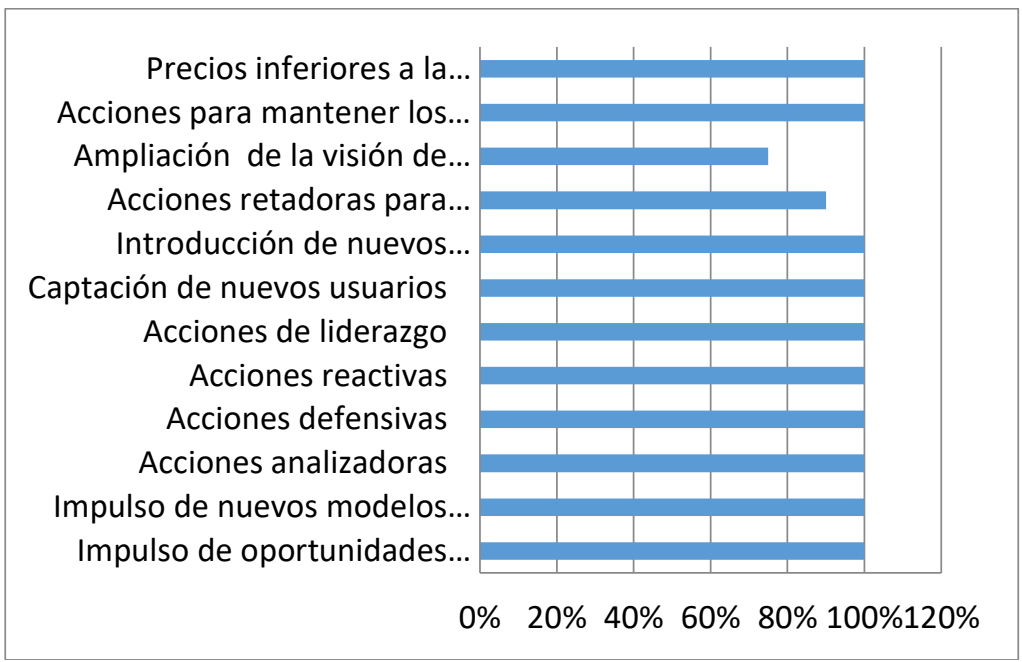

Fuente: Elaboración propia.

En términos generales el gráfico 2 sintetiza todas las acciones que corresponden a la estrategia competitiva en centros de especialización de odontología, que se esfuerzan por ser más eficaces que otros, para permanecer en su posición o mejorar la misma en su segmento de mercado. En este sentido, García et al. (2019) explican que estas estrategias requieren de una actitud gerencial estratégica en concordancia con el marketing para tener una orientación y direccionamiento de esfuerzos en común y con un único fin: intentar dominar el mercado.

A manera de síntesis, el cuadro 2 permite identificar las estrategias y acciones desarrolladas por los centros especializados de odontología, observando el predominio de acciones para crecer y competir en época pandémica.

Cuadro 2

Estrategias de Marketing Odontológico

\begin{tabular}{|c|c|c|}
\hline $\begin{array}{l}\text { Estrategias de } \\
\text { Diferenciación }\end{array}$ & Estrategias de Crecimiento & $\begin{array}{l}\text { Estrategias } \\
\text { competitivas }\end{array}$ \\
\hline $\begin{array}{c}\text { Bajar los } \\
\text { costos }\end{array}$ & $\begin{array}{c}\text { Nuevas generaciones de insumos } \\
\text { odontológicos }\end{array}$ & $\begin{array}{l}\text { Impulso de oportunidades de } \\
\text { innovación }\end{array}$ \\
\hline $\begin{array}{l}\text { Bajos los } \\
\text { precios }\end{array}$ & $\begin{array}{l}\text { Competencias/habilidades } \\
\text { de los odontólogos actualizadas }\end{array}$ & $\begin{array}{l}\text { Impulso de nuevos modelos de } \\
\text { negocios }\end{array}$ \\
\hline $\begin{array}{l}\text { Cualidades } \\
\text { distintivas }\end{array}$ & Inversión en nuevas tecnologías & Acciones analizadoras \\
\hline & $\begin{array}{l}\text { Acuerdos de cooperación y } \\
\text { alianzas estratégicas }\end{array}$ & Acciones defensivas \\
\hline
\end{tabular}




\begin{tabular}{|c|c|}
\hline $\begin{array}{c}\text { Incrementar de la cuota de } \\
\text { mercado }\end{array}$ & Acciones reactivas \\
\hline Nuevas promociones & Acciones de liderazgo \\
\hline Precios accesibles a la población & Captación de nuevos usuarios \\
\hline $\begin{array}{l}\text { Acciones comunicacionales } \\
\text { (publicidad digital) }\end{array}$ & $\begin{array}{c}\text { Introducción de nuevos } \\
\text { servicios }\end{array}$ \\
\hline $\begin{array}{c}\text { Nuevas generaciones de insumos } \\
\text { odontológicos }\end{array}$ & $\begin{array}{l}\text { Acciones retadoras para } \\
\text { superar las fortalezas de la } \\
\text { competencia }\end{array}$ \\
\hline & $\begin{array}{c}\text { Ampliación de la visión de los } \\
\text { servicios }\end{array}$ \\
\hline & $\begin{array}{c}\text { Acciones para mantener los } \\
\text { costos bajos }\end{array}$ \\
\hline & $\begin{array}{l}\text { Precios inferiores a la } \\
\text { competencia }\end{array}$ \\
\hline
\end{tabular}

Fuente: Elaboración propia.

En el cuadro 2 también se puede apreciar que las acciones referentes a bajos costos y bajos precios se encuentran presentes tanto en las estrategias de diferenciación como en las estrategias competitivas, ello indica que son acciones que su bi-direccionalidad tienen gran incidencia en el marketing odontológico de las organizaciones en estudio en el cantón de Ambato.

En el desarrollo de los resultados se ha podido apreciar que ha predominado en términos generales una tendencia positiva en tres tipos de estrategias de marketing orientadas a la diversificación, el crecimiento y la competitividad de los centros especializados de odontología; con ello se demuestra que a pesar que las dificultades sociales, temores de salud al contagio por el COVID-19 y turbulencia del contexto económico las mencionadas organizaciones de salud han estado a la vanguardia estratégica en el cantón de Ambato de la provincia de provincia de Tungurahua en Ecuador, para afrontar las vicisitudes y continuar desenvolviéndose en su segmento de mercado competitivamente.

\section{Conclusiones}

El desenvolvimiento de los centros especializados de odontología se ha visto afectado significativamente por el COVID-19 y aunado a la crisis socioeconómica que ha generado la pandemia, han tenido que afrontar nuevos retos de un entorno socioeconómico complejo e incierto. Para los profesionales de la odontología los desafíos son mayor, pues deben trabajar sabiendo que están expuestos a un alto riesgo de contagio, por tener que trabajar la salud y enfermedades bucales ante varios agentes patógenos, que pueden llegar a afectar la cavidad oral y el tracto respiratorio, y aunque se han acogido medidas de higiene y bioseguridad, tanto los odontólogos como los pacientes, el temor a enfermarse es latente, lo cual hace más difícil a este tipo de organizaciones mantenerse en condiciones óptimas de funcionamiento.

Teniendo presentes las dificultades antes mencionadas, las organizaciones, prestadores de servicio de salud tienen que estar a disposición de la sociedad; aunado a que los compromisos 
financieros, laborales y tributarios, entre otros, se encuentran vigentes y se deben responder a los mismos de manera oportuna, como estipula la normativa legal nacional y provincial, para no contraer multas o penalidades fiscales.

Cumplir con los compromisos y cubrir los costos de funcionamiento en una economía nacional y local deprimida ha sido una labor titánica, y ha conducido a estos centros de especialidades de odontología a implementar estrategias de marketing, para aprovechar las oportunidades del mercado que han surgido en tiempos de pandemia, procurar mantener sus usuarios/pacientes, captar usuarios potenciales e ir ampliando su participación en el mercado local.

En tal sentido, se ha encontrado un alto dinamismo de estos centros de salud, pues conscientes de los retos que deben enfrentar, también han dirigido que de manera oportuna sus recursos hacia la diferenciación, crecimiento y competitividad, procurando captar beneficios, mejorar su posicionamiento en el mercado e identificar nuevas alternativas en este difícil escenario sanitario, económico y social, así como potenciar directa e indirectamente las gestiones de la mezcla de marketing en los mencionados centros su actividad.

Se espera que este trabajo sea un abreboca a futuras investigaciones del marketing en este campo de salud, estimulando a profundizar sobre el marketing estratégico, y correlacionarlo con otros temas, como: la imagen corporativa, marketing experiencial o marketing transcultural, entre otros.

\section{Referencias Bibliográficas}

Aguilera-Castro, Adriana; Ávila-Fajardo, Gloria y Solano-Rodríguez, Omar. (2017). Las TIC en la formulación estratégica de las pymes de Santiago de Cali-Colombia. Entramado, 13(1). Colombia https://doi.org/10.18041/entramado.2017v13n1.25106

Ansoff, Igor. (1965). Corporate Strategy. Editorial McGraw-Hill. USA.

Arboleda, Oscar; Baptista, Dulce; González-Velosa, Carolina; Novella, Rafael; Rosas-Shady, David; Silva Porto, María Teresa y Soler, Nicolás. (2020). ¿Cómo proteger los ingresos y los empleos?: Posibles respuestas al impacto del coronavirus (COVID-19) en los mercados laborales de América Latina y el Caribe. Banco Interamericano de Desarrollo DOI: http://dx.doi.org/10.18235/0002307

Arellano, Rolando. (2010). Marketing: Enfoque América Latina. Pearson Educación. México. Ariño, África (2007). Alianzas estratégicas: opciones para el crecimiento de la empresa (I). Estrategia Financiera, 236. España (Pp. 40-51). Extraído de: http://pdfs.wke.es/6/8/6/2/pd0000016862.pdf

Best, Roger. (2007). Marketing Estratégico. Editorial Pearson Prentice Hall. España.

Canals, Jordi. (2001). How to think about corporate growth? European Management Journal, 19 (6). USA. (587-598). DOI: https://doi.org/10.1016/S0263-2373(01)00084-6

Chandler, Alfred. (1962). Strategic and structure: chapters in the history of american industries enterprise. MIT Press. Inglaterra.

Comisión Económica para América Latina y el Caribe. (2020). Informe especial dimensionar los efectos del covid-19 para pensar en la reactivación. Extraído de: https://repositorio.cepal.org/bitstream/handle/11362/45445/4/S2000286_es.pdf 
Comité Empresarial Ecuatoriano (2020). Oficio al IESS debido a los impactos de la crisis sanitaria en el sector productivo en ámbitos como la liquidez, el empleo, entre otros. Extraído de http://cee.org.ec/wp-content/uploads/2020/04/Carta-IESSFederaciones-170420final.pdf

Cordero, Paula; Chavarría, Hugo; Echeverri y Sepúlveda, Sergio. (2003). Territorios rurales, competitividad y desarrollo. IICA. Costa Rica. Extraído de: https://www.researchgate.net/publication/237835428_TERRITORIOS_RURALES COMPETITIVIDAD_Y DESARROLLO

Demuner Flores, María. (2021). Uso de redes sociales en microempresas ante efectos COVID19. Revista de Comunicación de la SEECI, (54). España (Pp.97-118). DOI: https://doi.org/10.15198/seeci.2021.54.e660

Dental Tribune Latin América (2017). Henry Schein completa la adquisición de Dental Cremer. Extraído de: https://n9.cl/6yms4

El Universo (2021). Desde este miércoles en Ambato regirá nuevo horario de restricción vehicular. Extraído de: https://www.eluniverso.com/noticias/ecuador/desde-estemiercoles-21-en-ambato-regira-nuevo-horario-de-restriccion-vehicular-notal

Ferrel, Oscar y Hartline, Michael (2012). Estrategia de Marketing. Quinta Edición. Cengage Learning Editores. México.

Furrer, Olivier; Thomas, Howard y Goussevskaia, Anna (2007). The structure and evolution of the strategic management field: A content analysis of 26 years of strategic management research. International Journal of management Reviews, 10(1). USA. (Pp. 1-23). DOI: https://doi.org/10.1111/j.1468-2370.2007.00217.x

García, Jesús; Paz, Annherys y Cantillo, Nair. (2019). Estrategia y habilidades para la competitividad: caso de pymes del sector construcción en Barranquilla. Revista
Aglala
10
(1).
Colombia.
(Pp.
312-229).

DOI: https://doi.org/10.22519/22157360.1349

Hill, Brendan. (2020). Marketing During Times of Uncertainty: Advice from Marketing Experts. Extraído de: https://metigy.com/metigy-learning/marketing-during-times-ofuncertainty-advice-from-marketing-experts/.

Hitt, Michel; Ireland, Duane y Hoskisson, Robert. (2008). Administración Estratégica. $7^{\circ}$ Edición. Cengage Learning Editores, S.A. México.

Kotler, Phillip y Armstrong, Gary (2007). Marketing. Versión para Latinoamérica. Editorial Prentice Hall. México.

Lambin, Jean Jacques; Galluci, Carlo y Sicurello, Carlos (2009). Dirección de marketing. Gestión estratégica y operativa del mercado. McGraw-Hill. México.

Medina, Alex; Constanzo, Alexis y Sandoval, Rodrigo (2012). Estrategias de diversificación y concentración empleadas por las sociedades anónimas en Chile. Contaduría y Administración, 57 (4). México. (Pp. 55-77). Extraído de: http://www.scielo.org.mx/pdf/cya/v57n4/v57n4a4.pdf

Miles, Raymond y Snow, Charles (1978) Organizational strategy, structure and process. McGraw-Hill. USA.

Miles, Raymond; Snow, Charles; Meyer, Alan y Coleman Henry. (2003). Organizational Strategy, Structure and Process. The Academy of Management Review, 3 (3). USA. (Pp. 546562). Extraído de: http://www.jstor.org/stable/257544 
Miller, Peneelope Ann. (1987). Relaciones industriales estratégicas y gestión de recursos humanos - distinción, definición y reconocimiento. Journal Managment Sudies, 24 (4). USA (pp. 347-361). DOI: https://doi.org/10.1111/j.1467-6486.1987.tb00450.x Mintzberg, Henry; Ahlstrand, Bruce y Lampel, Joseph (1998). Strategy Safari: A Guided Tour Through. The Wilds of Strategic Management. The free Press. USA. Extraído de: https://www.academia.edu/1895549/Strategy_safari_A guided tour through the wil ds of strategic management

Molina, Olga. (2003). Nuevas Técnicas de Control y Gestión de Costos en Búsqueda de la Competitividad Actualidad Contable Faces, 6(6). Venezuela. (Pp. 25-32). Extraído de: https://www.redalyc.org/pdf/257/25700604.pdf

Monferrer, Diego. (2013). Fundamentos de marketing. Universitat Jaume I. España. Extraído de: http://repositori.uji.es/xmlui/bitstream/handle/10234/49394/s74.pdf

Moreno, Lenin (2020). Decreto de Estado de Emergencia Sanitaria. No 1017. Extraído de: https://www.defensa.gob.ec/wp-

content/uploads/downloads/2020/03/Decreto_presidencial_No_1017_17-Marzo2020.pdf

Moscoso-Moscoso, Francisco; Rivera-Costales, José;González-Rodríguez, Ramiro y MatovelleRomo, Moisés (2020). Marketing de guerrilla aplicado a empresas del sector de equipamiento dental. Revista Arbitrada Interdisciplinaria KOINONIA, 5 (3). Venezuela (pp. 805-834). http://dx.doi.org/10.35381/r.k.v5i3.922

Mullins, John; Walker, Orville; Boyd, Harper y Jamieson, Barbara (2013). Marketing. Edinburgh Business School. Inglaterra.

Munuera, José y Rodríguez, Ana. (2012). Estrategias de Marketing. Editorial ESIC. España. Muñiz, Rafael. (2012). Marketing en el siglo XXI. Cuarta edición, Editorial CEF. Colombia.

Extraído

http://pdfi.cef.es/marketing_en_el_siglo_xx1_freemium/files/assets/common/downlo ads/publication.pdf

Navarro, Beatriz. (2021). El liderazgo en tiempos de pandemia: cambios y nuevas tendencias para el siglo XXI. Extraído de:

http://www.ieee.es/Galerias/fichero/docs_opinion/2021/DIEEEO14_2021_BEANAV LidPan.pdf

Parise-Vasco, Juan; Zambrano-Achig, Paula; Viteri-García, Andrés y Armas-Vega, Ana (2020). Estado de la salud bucal en el Ecuador. Odontol. Sanmarquina. 23(3). Perú. (Pp.327-332). DOI: https://doi.org/10.15381/os.v23i3.18133

Porter, Michael. (1980). Competitive strategy. Techniques for analyzing industries and competitors. The Free Press. USA.

Porter, Michael. (1985). Estrategia Competitiva: Técnicas para el análisis de los sectores industriales y de la competencia. Editorial Continental, México.

Primicias. (2021). Banco Mundial: economía de Ecuador crecerá 3,4\% en 2021. Extraído de: https://www.primicias.ec/noticias/economia/banco-mundial-economia-ecuador2021/

Real, Isidro, Leyva; Alma y Heredia, José. (2018). Uso e impacto de las redes sociales en las estrategias de marketing de las PyME's. Revista de Investigación Académica Sin Frontera, $7(19)$. México.

(Pp.1-24). Extraído de 
https://revistainvestigacionacademicasinfrontera.unison.mx/index.php/RDIASF/articl e/view/47/46

Rubio, Luis y Baz, Verónica. (2015). El poder de la competitividad. DIDAC. México. Extraído de: http://www.cidac.org/esp/uploads/1/Competitividad_FINAL-Agosto_2004.pdf

Sigua-Rodríguez, Eder Alberto; Bernal-Pérez, Jorge Luis; Lanata-Flores, Antonio Gabriel; Sánchez-Romero, Celeste;Rodríguez-Chessa, Jaime;Haidar, Ziyad S;Olate, Sergio, y Iwaki-Filho, Liogi. (2020). COVID-19 y la Odontología: una Revisión de las Recomendaciones y Perspectivas para Latinoamérica. International journal of odontostomatology, 14(3). Chile. (Pp. 299-309). DOI: https://dx.doi.org/10.4067/S0718-381X2020000300299

Suárez Salgado, Sandra; Campuzano, Roberto; Dona Vidale, Marina; Garrido Cisneros, Eduardo y Giménez Miniello, Thais (2020). Recomendaciones para prevención y control de infecciones por SARS-CoV-2 en odontología. Revista Odontología, 22 (2), Ecuador. (Pp. 5-32). https://doi.org/10.29166/odontologia.vol22.n2.2020-5-32

Superintendencia de Compañías, Valores y Seguros de Ecuador (2019). Panorama de la inversión empresarial en el Ecuador Período: 2013 - 2018. Extraído de: https://n9.cl/q4pmh

Tarazona, Ariel. (2020). Relaciones en tiempos de pandemia: COVID-19 y bienestar animal, ambiental y humano. Revista Facultad Nacional de Agronomía, 73 (2). Colombia. (Pp. 1-3). https://doi.org/10.15446/rfnam.v73n2.86957

Useche, María; Vásquez Lacres, Lourdes Maribel; Salazar Vázquez, Flor Isabel; Ordóñez Gavilanes, Magdalena (2021). Fórmula Estratégica Empresarial para Pymes en Ecuador ante el Covid-19. Universidad \& Empresa. 23 (40). Colombia. (Pp.1-22). DOI: https://doi.org/10.12804/revistas.urosario.edu.co/empresa/a.9309

Villagómez, Alan y Acosta, Elizabeth. (2020). Uso de redes sociales digitales como estrategia de mercadotecnia en pymes pirotécnicas de Tultepec en el Estado de México. Revista de Comunicación de la SEECI, (52). España. (Pp. 73-93). http://www.seeci.net/revista/index.php/seeci/article/view/610.

Walker, Orvillec; Boyd, Harper; Mullins, John y Larreche, Pierre. (2005). Administración de Marketing. Un enfoque en la toma estratégica de decisiones. $5^{\circ}$ Edición. Editorial Mc. Graw Hill Interamericana. México.

Whittington, Richard. (2003). Qué es la estrategia? ¿Realmente importa?. Tompson editores. España. 